\title{
Video Feedback on Mother-child Interactions in Perinatal Psychiatric Service in Hong Kong
}

Maternal mood disorders have an adverse effect on parenting and childhood development. A meta-analysis of 46 observational studies demonstrated a moderate association between maternal depression and negative parenting behaviour, and a small-to-moderate effect on disengaged behaviour. ${ }^{1}$ In particular, maternal depression is associated with two parenting patterns (hostile or intrusive and disengaged or withdrawn) that affect childhood development. ${ }^{1}$ Treatment of postnatal depression with mother-child intervention benefits both children and mothers. ${ }^{2}$ A good quality perinatal mental health service should include parenting interventions. ${ }^{3}$ In the United States and Australia, parenting interventions are routinely incorporated into the perinatal and infant mental health service. In Hong Kong, the Comprehensive Child Development Service provides service to high-risk pregnant women and mothers with postnatal depression, with the goal to improve maternal mental health and foster healthy child development (until 5 years of age). Parenting interventions are an important element of the overall clinical management of depressed mothers by the perinatal psychiatric service in Hong Kong.

Circle of Security ${ }^{4}$ and Tuning Into Kids ${ }^{5}$ programmes have been used as therapeutic group interventions in the Comprehensive Child Development Service to promote parental responsiveness and positive parenting. Nonetheless, for those who are unwilling to participate in groups or whose emotions are too fragile such that group intervention is inappropriate, video feedback is an alternative option that has been shown to improve parent-child interactions in the short-term, especially in depressed parents and parents with problematic interactions, and to have a long-term positive effect on child social/emotional development and depressive symptoms of parents. ${ }^{6}$ Even a single psychotherapy session with video feedback of a scene of mother-child separation results in a significant reduction in negative perception in mothers traumatised by family violence. ${ }^{7}$ The process increases parental sensitivity and enhances attachment security, ${ }^{8}$ and may change parental representations of the child or parent-child interaction patterns or both. ${ }^{6}$

At the Pamela Youde Nethersole Eastern Hospital in Hong Kong, video feedback has been incorporated into the Comprehensive Child Development Service in the Perinatal Psychiatric Clinic since October 2016. Video feedback is delivered as an adjunctive treatment to mothers with postnatal mood disorders whose preschool children present with emotional/behavioural problems (frequent temper tantrums, exaggerated separation anxiety, aggressive behaviours), those with harsh/coercive/disengaged parenting, and those unwilling or unsuitable to join a group programme and whose parenting behaviour has not been improved by individual counselling. Mothers who receive the intervention have postnatal depression or adjustment disorder, with mild to moderate depressive symptoms. The goal of video feedback is to improve parental sensitivity and attachment security, as well as to enhance motivation to change parenting behaviours. A 30-minute video captures mother-child interactions in a play room in the clinic with age-appropriate toys, while the therapist observes through a one-way mirror. The session includes the Strange Situation Procedure, ${ }^{9} 3$ minutes of reading time, and 3 minutes of tidying up toys. The two authors analyse the video and evaluate the attachment security (using the Strange Situation Procedure ${ }^{1}$ ) and parental sensitivity (how engaged or responsive the mother is to her child; whether she can follow the child's lead to support play and exploration; whether she is too intrusive during play time). We also observe how well the mother can comfort the child after separation and the child's response. A 30-minute feedback session is then provided one week later. Segments of video, each lasting 1 to 2 minutes, are selected to show the mother her strengths and weaknesses in the interaction. The whole approach is strength-based, with the aim to help the mother reflect on her own interactions with the child from a different perspective, without overwhelming the mother's emotions.

In six of the mother-child dyads, we made a further video after 6 months with no parenting intervention provided in between. In three of the dyads, the attachment security changed from insecure to secure, one from insecure disorganized to insecure avoidant and the remaining two had secure attachment in the beginning and remained so 6 months later, with increased maternal responsiveness to the child. Using the Parenting Sense of Competence Scale, there was a trend towards increased parental efficacy after 6 months as shown by an increase in the mean efficacy score from 22.33 to $26.00(\mathrm{t}=-2.17, \mathrm{p}=0.082)$, although the sample size was too small to achieve statistical significance.

Our initial experience suggests that video feedback may be useful in a perinatal psychiatric setting in Hong Kong. Although the intervention was not conducted in a research setting and the sample size was too small for meaningful interpretations, the initial results suggest that video feedback has a role in improving mother-child interactions, particularly in those who do not recognise their problems. The video feedback allows the mothers to watch themselves and reflect upon their interactions objectively. It enhances parental mentalisation capacities and motivates mothers to change their parenting behaviours. Video feedback can be a good alternative when participation in 
parenting groups is inappropriate, or when individual counselling sessions become back-and-forth arguments about what constitutes proper parenting behaviours. Further research about the usefulness of video feedback in a perinatal psychiatric service in Hong Kong is needed to determine its effectiveness and the number of sessions needed, while balancing the costs.

Dr Pui Yin Joseph Chung, MRCPsych, FHKCPsych, FHKAM(Psychiatry) (email: chungpy2@ha.org.hk)

Associate Consultant, Comprehensive Child Development Service, Department of Psychiatry, Pamela Youde Nethersole Eastern Hospital, Hong Kong

Ms Wai Yin Iris Wong, M Soc Sc in Clinical Psychology (CUHK)

Clinical Psychologist, Comprehensive Child Development Service, Kwai Chung Hospital, Hong Kong

\section{References}

1. Lovejoy MC, Graczyk PA, O’Hare E, Neuman G. Maternal depression and parenting behavior: a meta-analytic review. Clin Psychol Rev 2000;20:561-92. Eross ref

2. Poobalan AS, Aucott LS, Ross L, Smith WC, Helms PJ, Williams JH. Effects of treating postnatal depression on mother-infant interaction and child development: systematic review. Br J Psychiatry 2007;191:37886. kross re

3. Guidance for commissioners of perinatal mental health services. Joint Commissioning Panel for Mental Health, UK. 9 December 2012.

4. Website: https://www.circleofsecurityinternational.com/

5. Website: http://www.tuningintokids.org.au/

6. Hoivik MS, Lydersen S, Drugli MB, Onsoien R, Hansen MB, Nielsen TS. Video feedback compared to treatment as usual in families with parent-child interactions problems: a randomized controlled trial. Child Adolesc Psychiatry Ment Health 2015;9:3. cross re

7. Schechter DS, Myers MM, Brunelli SA, Coates SW, Zeanah CH, Davies M, et al. Traumatized mothers can change their minds about their toddlers: understanding how a novel use of videofeedback supports positive change of maternal attributions. Infant Ment Health J 2006;27:429-47. Eross re

8. Bakermans-Kranenburg MJ, van IJzendoorn MH, Juffer F. Less is more: meta-analyses of sensitivity and attachment interventions in early childhood. Psychol Bull 2003;129:195-215. Eross re

9. Ainsworth MD, Blehar MC, Waters E, Wall S. Patterns of Attachment: A Psychological Study of the Strange Situation. 1st ed. Psychology Press; 2015. 